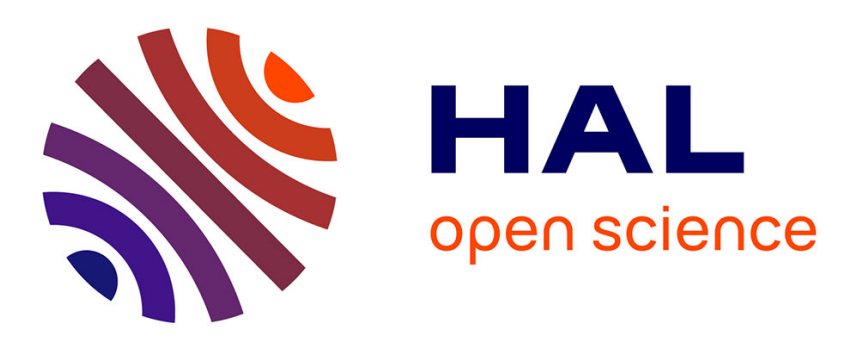

\title{
Rydberg Atoms And Radiation In A Resonant Cavity II. Experiments
}

\author{
C. Fabre, S. Haroche, J. Raimond, P. Goy, M. Gross, L. Moi
}

\section{To cite this version:}

C. Fabre, S. Haroche, J. Raimond, P. Goy, M. Gross, et al.. Rydberg Atoms And Radiation In A Resonant Cavity II. Experiments. Journal de Physique Colloques, 1982, 43 (C2), pp.C2-275-C2-283. 10.1051/jphyscol:1982221 . jpa-00221832

\section{HAL Id: jpa-00221832 https://hal.science/jpa-00221832}

Submitted on 1 Jan 1982

HAL is a multi-disciplinary open access archive for the deposit and dissemination of scientific research documents, whether they are published or not. The documents may come from teaching and research institutions in France or abroad, or from public or private research centers.
L'archive ouverte pluridisciplinaire HAL, est destinée au dépôt et à la diffusion de documents scientifiques de niveau recherche, publiés ou non, émanant des établissements d'enseignement et de recherche français ou étrangers, des laboratoires publics ou privés. 
JOURNAL DE PHYSIQUE

Colloque C2, supplément au $n^{\circ} 11$, Tome 43, novembre 1982

page $\mathrm{C} 2-275$

\section{RYDBERG ATOMS AND RADIATION IN A RESONANT CAVITY II. EXPERIMENTS}

C. Fabre, S. Haroche, J.M. Raimond, P. Goy, M. Gross and L. Moi

Laboratoire de Physique de l'Ecole Normale Supérieure, 24 me Lhomond, 75231 Paris Cedex 05, France

Résumé. - Nous prësentons diffërentes expériences mettant en jeu des atomes de

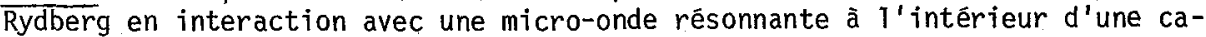
vitē, permettant de vérifier diffërents aspects de 1a théorie présentée dans †'exposế précédent. Nous décrivons d'abord des expériences d'émission superradiante des états de Rydberg dans la cavitê ( effet maser transitoire à atomes de Rydberg!̣. L'évolution du système est détectée soit sur les populations par la technique d'ionisation sélective par champ, soit sur le champ microonde par détection hëtérodyne. Des seuils très bas de. 7 'émission maser, jusqu' à 100 atomes, ont étê observés. Nous décrivons ensuite quelques aspects de 7 'absorption collective de rayonnement par les atomes trēs excités, en particuTier Trobservation de la nutation de Rabi sous ' 'effet d'un faible champ extérieur, l'étude du rendement quantique de notre système considéré comme un compteur de photons millimétriques et enfin la mise en évidence d'aspects inattendus dans ''absorption de rayonnement du corps noir dans la cavité.

Abstract. - We present various experiments involving Rydberg atoms interacting with resonant microwave electromagnetic fields inside a cavity, allowing to check different features of the theory presented in the previous paper. We first describe experiments about superradiant emission by Rydberg states in the cavity, also called transient Rydberg atoms maser; the evolution of the system is detected either on the populations by selective field ionization technique or on the microwave field itself by heterodyne detection. Very low thresholds, down to 100 atoms, for the maser emission have been observed. We then describe various aspects of collective absorntion of radiation by very excited atoms including the observation of Rabi nutation in a small applied resonant field, the study of the quantum efficiency of this set-up considered as a microwave photon counter, and finally the unexpected characteristics of the blackbody radiation absorption in the cavity.

In this second paper, we present various experiments on Rydberg states interacting with resonant millimetric electromagnetic waves inside a cavity. These experiments show various interesting physical effects calculated in the previous paper and provide a test for the theoretical models. In a first paragraph, we will describe the experimental techniques which are common to all the experiments that will be presented afterwards. We will devote the second paragraph to an experimental study of the different characteristics of the collective Rydberg atom transient emission in the cavity (the "Rydberg atom maser"). In the last paragraph, we will describe several experiments involving the absorption of radiation by very excited states in the cavity and showing the collective behaviour of the Rydberg atoms in this absorption process.

\section{Experimental techniques}

\subsection{Preparation of the_physical__system}

In order to prepare the physical system which has been theoretically studied in the previous paper, i.e. a set of 2-level. Rydberg atoms in resonant interaction with an electromagnetic mode inside a cavity, we use the experimental set-up which 
is schematized on Figure 1 :

- Two simultaneous laser pulses populate, by a stepwise excitation, a given Rydberg state $\mid n S>(n \approx 30)$ of alkalj atom (sodium or cesium) in a thermal beam. The number $N$ of very excited states created by each laser pulse can be varied from 1 to $10^{6}$. Due to the long 7 ifetimes of such states (several tens of $\mu 5$ ), they drift at thermal velocities along the atomic beam over several centimeters before decaying to lower lying states.

- The Rydberg atoms are created in an antinode of the standing wave of the Gaussian mode

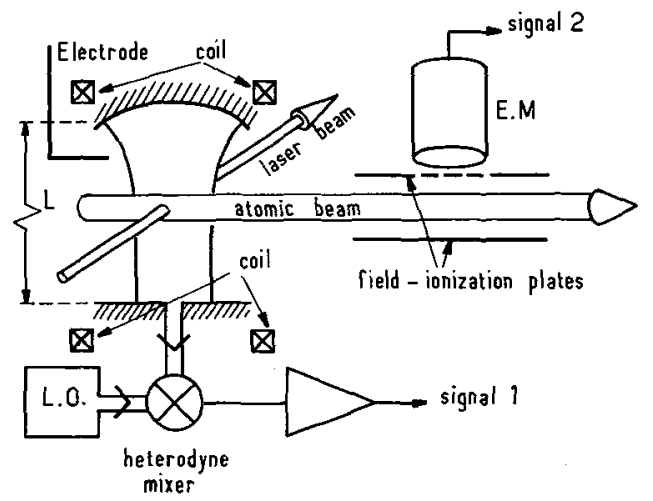

Figure 1: Scheme of the experimental set-up

existing in a semi-confocal Fabry-Perot cavity, the axis of which is perpendicular to the atomic beam. The resonant frequency of the cavity can be adjusted by varying the distance $L$ between the two mirrors. The cavity typical characteristics are the following: length $L: \sim 1 \mathrm{~cm}$; waist $w_{\circ}$ of the Gaussian mode : $\sim 3 \mathrm{~mm}$; volume $V$ of the mode in the cavity : $20.1 \mathrm{~cm}^{3}$; quality factor $Q$ around $150 \mathrm{GHz}$ : $10^{4}$; damping time of the mode in the cavity $Q / \omega=\mathrm{T}_{\text {cav }} \sim 9$ ns (which is much less than the transit time $T \sim 3 \mu \mathrm{s}$ of the atoms inside the Gaussian mode). The cavity can be fed through a wave guide by an external $\mathrm{mm}$ source (carcinotron or frequency multiplied klystron).

- For most experiments, the cavity is tuned to the Bohr frequency of the transition $n S^{1 / 2} \rightarrow n^{\prime} P^{1} / 2\left(n^{\prime}=n\right.$ or $\left.n-1\right)$, so that two transitions $\mid n S^{1 / 2} m_{J}=+1 / 2>\rightarrow$ $\mid n^{\prime} \mathrm{P} 1 / 2 \mathrm{~m}_{\mathrm{J}}=-1 / 2>$ and $\left|\mathrm{nS}^{1} / 2 \mathrm{~m}_{\mathrm{J}}=-1 / 2>\rightarrow\right| \mathrm{n}^{\prime} \mathrm{P} 1 / 2 \mathrm{~m}_{\mathrm{J}}=+1 / 2>$ interact independent$1 y$ with the $\sigma_{+}$and $\sigma_{-}$polarizations of the electromagnetic field mode inside the cavity (the "quantization" $O z$ axis being chosen along the cavity axis). For some experiments, it has been necessary to study a unique two-level system interacting with the cavity mode. For that purpose, two coils coaxial to the cavity can be used to apply a static magnetic field $B_{0}$ along the $z$-axis and split apart the two Zeeman components of the transition. If the field is large enough $\left(B_{0} \geqslant\right.$ $50 \mathrm{G}$ ), the Zeeman splitting $\approx 100 \mathrm{MHz}$ is larger than the width of the cavity mode, so that the cavity can be tuned to resonance with a single two-level-atom system.

\subsection{Detection of the evolution}

Two techniques have been used to detect the evolution of the system atom + field, the first one on the field, and the second on the atoms :

(i) To monitor the electromagnetic field at the output of the cavity, we use an heterodyne detector $\left({ }^{1}\right)$ (signal 1 in Fig. 1) : it consists in a low noise Schottky diode which mixes the signal to measure and the output of a local oscillator (stabilized carcinatron). The low frequency beatnote ( $100 \mathrm{MHz}$ ) is then amplified, filtered, monitored, and eventualiy rectified and averaged over several laser shots. This technique allows us to record the whole evolution of the system for each laser pulse without perturbation; but is restricted 
to rather "intense" signals (number $N$ of excited atoms $\geqslant 10^{5}$ which corresponds to a signal of $10 \mathrm{pW}$ during a few hundreds nanoseconds). Furthermore, the narrow bandpass filter used to reduce the thermal noise on the beat signal limitates the time response of the detection to roughly $80 \mathrm{~ns}$.

(ii) To detect the evolution of the Rydberg level poputations, we make use of the

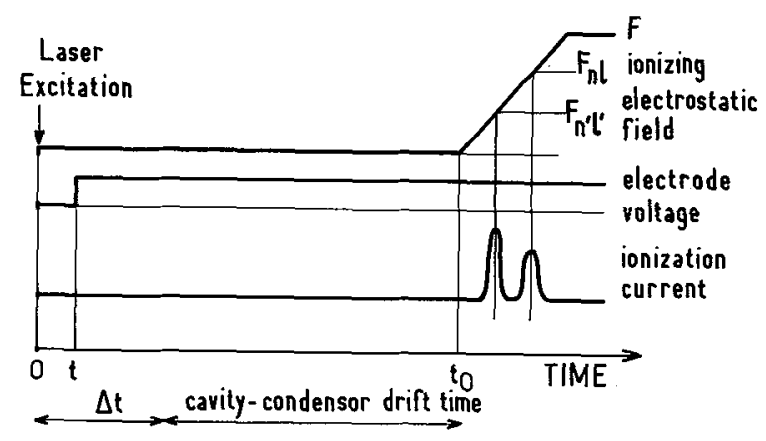

Figure 2 : Time sequence of different experimental parameters. $\Delta t$ is the time of filight of the atoms in the cavity mode. The atom-cavity interaction is stopped at time $t$ by the voltage applied on the electrode. The ramp of electric field applied from time $t_{0}$ ionizes at different times the different Rydberg levels. The resulting ionization current displays peaks corresponding to the different Rydberg states populated at time $t_{0}$.

now well-known selective electrostatic field ionization technique (signal 2 in Fig. 1) : at the exit of the Fabry-Perot cavity, the atoms enter a plane condenser inside which an electrostatic field $F$ is applied. Electrons created by ionization at the center of the condenser can be detected through a mesh by an electron-multiplier. Figure 2 shows the sequence of events for each 1 aser pulse : at time $t=0$, the Rydberg atoms are created inside the cavity and interact with the electromagnetic field mode during a time $\Delta t$. They drift afterwards in the condenser and are submitted from time to to a ramp of electric field $F(t)$. The thresholds $F_{n S}$ and $F_{n ' p}$ are reached at slightly different times. The ionisation current exhibits two peaks as a function of time, the areas of which yield the populations of the levels $n S$ and $n^{\prime} P$ at time $t_{0}$ (when the gain of the e.m. is known). Since the effective lifetimes and the thermal transfer rates of the two levels are known, we can deduce the populations just after the end of the interaction between the atoms and the cavity. To know the populations of the two levels at any time $t<\Delta t$ during the evolution inside the cavity, we use the following method: at time $t$, a fast rising voltage is applied between an electrode E (see Fig. 1) and the grounded cavity : this voltage induces an inhomogeneous Stark shift such that the transition frequency between levels nS and $n^{\prime} P$ is no longer resonant with the cavity : the interaction atoms-cavity stops at this time $t$ and the system is "frozen" in its evolution. By varying $t$ we can sample the evolution of the atomic system.

The whole detection system is interfaced with a computer LSI11 which computes on line the populations of the levels, and in order to get rid of the pulse-to-pulse fluctuations of the laser excitation, records only the ionization signal corresponding to a given number $N$ (within a few \%) of initially excited Rydberg levels. This sampling technique is very convenient and can be used even for very low $\mathrm{N}$ numbers of excited atoms, since the field ionization detection can count one by one the Rydberg atoms arriving inside the condensor. 
2. Experimental study of Rydberg state superradiance in a cavity (Rydberg masers)

\subsection{General characteristics $\left({ }^{2}\right)\left({ }^{3}\right)\left({ }^{4}\right)\left({ }^{5}\right)$}

A first class of experiments consists in exciting $N$ Rydberg atoms in an InS $>$ state inside the cavity and in tuning the cavity to resonance with a transition linking this state to a lower lying $\mid n^{\prime} p>$ state. Usually $n^{\prime}=n-1$, i.e. $n^{\prime} P$ is the closest $P$ level of larger binding energy, but it can be also $n-2, n-3$ in some cases. In the Bloch vector representation, it corresponds to an initial Bloch angle $\theta=0$. If $N$ is large enough, the superradiant decay to the $\theta=\pi$ final state can occur before the atoms leave the cavity. The energy of the cavity electromagnetic mode is then temporarily increased by the coupling with the excited atoms : such an effect can be named transient Rydberg state maser. According to the actual characteristics of the cavities we have used, we have been always, so far, in the situation where $\omega / Q>\mathrm{T}_{\mathrm{R}}^{-1}$, which corresponds, according to the discussion of paragraph 6 in the

previous paper, to an overdamped, non oscillatory, transfer of population to the final $P$ state. 'The microwave energy $I(t)$ is emitted by the system in a brief burst of radiation, following the well known hyperbolic secant form :

$$
I(t)=\left[2 T_{R} \cos h\left(\frac{t-t_{D}}{2 T_{R}}\right)\right]^{-2}
$$

with $t_{D}$, delay time of the maser pulse, given by equation (10) of the previous paper.

Two different detection techniques we have described in the previous paragraph can be used to obtain different informations on this physical system :

(i) The detection of the final populations of the two levels involved in the transition by field ionization technique has provided the first experimental evidence for the Rydberg state maser and yields valuable informations on the thresholds $\mathrm{N}_{S}$ of the maser action. Figure 3 displays the ionization current delivered by

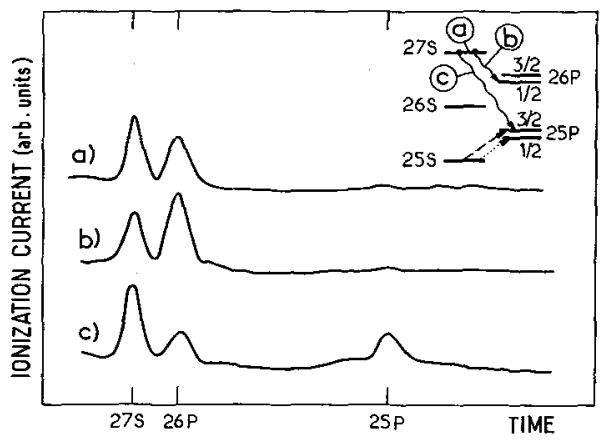

Figure 3 : Ionization signal showing maser action on different transitions when 275 state has been initially populated : a) untuned cavity; b) cavity tuned on $27 \mathrm{~S}-26 \mathrm{~S} 1 / 2$ transition;

c) cavity tuned on $27 \mathrm{~S}-25 \mathrm{P} 3 / 2$ transition

the electron multiplier as a function of time for different tuning positions of the cavity. The $27 \mathrm{~S}$ level has been initially populated by the laser. Trace a) corresponds to an untuned cavity : we see the peaks corresponding to levels 275 (populated by the 1aser) and 26P (populated by thermal background outside the cavity during the transit time between excitation and detection. Trace b) corresponds to a cavity tuned on the $27 \mathrm{~S}-26 \mathrm{P} 1 / 2$ transition frequency $(\lambda=1.47 \mathrm{~mm})$ and exhibits a strong enhancement of the $26 \mathrm{P}$ population, characteristic of the maser emission on this transition. When the cavity is now tuned to the $27 \mathrm{~S}-25 \mathrm{P} 3 / 2$ transition frequency 
$(\lambda=0.466 \mathrm{~mm})$, we observe a new peak on the ionization signal, corresponding to the $25 \mathrm{P}$ level. By this technique, we have put in evidence maser action on a large number of transitions between Rydberg states, ranging from $135 \mathrm{GHz}$ to $1.44 \mathrm{THz}$.

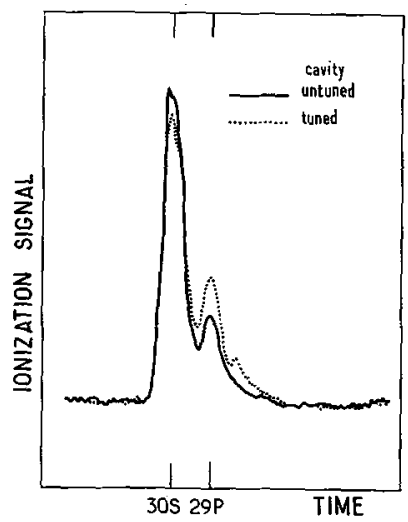

Fiqure 4 : Ionization signal showing maser action on 30 S - 29P3/2 transition in a high finesse cavity with only $100( \pm 50)$ excited atoms.

By determining the minimum number of excited atoms which yield an observable cavitydependent population transfer, we can measure the threshold for such a maser emission. This threshold can be shown to be inversely proportional to the finesse $f=Q\left(\frac{\lambda}{2 L}\right)$ of the cavity. Figure 4 shows a Rydberg atom maser near the threshold in a highly reflective cavity $(f=1600)$ : a difference in the population transfers between levels $30 \mathrm{~S}$ and $29 \mathrm{P} 3 / 2(\nu \approx 145 \mathrm{GHz})$ is clearly observable for a number of excited atoms as low as $100^{2}( \pm 50)$. These very low thresholds are characteristics for maser action between Rydberg states and are to be compared to the thresholds of the order of $10^{9}$ atoms for usual masers in the same frequency domain. By improving further the cavity mirrors reflectivity, we hope we will be able to obtain a collective emission of radiation by a very small number of Rydberg atoms, of the order of 1, for which the "single atom effects", outiined in paragraph 3 of the previous paper will be observable.

(ii) The detection by heterodyne techniques of the microwave signal emit-. ted by the atoms has the advantage of giving the pulse shape and the delay of the emission. The left part of figure 5 gives an example of such measurements, corres-
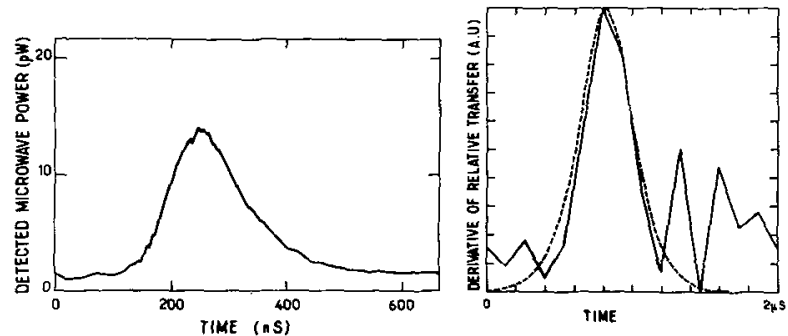

Figure 5 : Time evolution of the maser pulse : a) detected on the microwave by heterodyne mixing technique; b) detected by the "frozen evolution $^{11}$ field ionization technique. 
ponding to a peak intensity of $10 \mathrm{pW}$, delivered during a time of $100 \mathrm{~ns}$. The time labelled 0 corresponds to the laser excitation time. The pulse shape and the average delays measured by this way can be shown to be in good agreement with the theoretical predictions $\left({ }^{4}\right)$.

(iii) The right part of Figure 5 gives a typical signal recorded by the computer with the "frozen evolution" field ionization detection technique. The $y$-axis of this figure is in fact the time derivative of the population transfer, which is proportional to the field emitted by the atoms. This signal recorded on laser shots gives actualiy an average evolution of the maser corresponding to a given $N$ value of excited atoms. It exhibits a bell-shaped behaviour typical of superradiance, and has been recorded with $\mathrm{N}=50.000$ on $1 \mathrm{y}$ on the $30 \mathrm{~S} \rightarrow 28 \mathrm{P} 1 / 2$

transition ( $\nu=457.5 \mathrm{GHz}$ ).

\subsection{Experimental_study_of the maser_emission_statistics}

A specific feature of the Rydberg state maser is the large pulse to pulse variations of the maser pulse characteristics, even when the number of initially excited atoms is kept constant. As explained in the previous paper, these fluctuations are nothing else but the amplification of the photon fluctuations during the early stages of the maser evolution from its unstable initial state. The "frozen evolution" field ionization detection technique, described in $\S 1.2$, has enabled us to study experimentally the statistics of these fluctuations during the whole evolution of the system, and to quantitatively check with a good accuracy the formula (11) of the previous paper, giving the probability $P(n, t)$ of emitting $n$ photons between times 0 and $t$. The corresponding results wilt be presented in details elsewhere.

\section{Experimental study of resonant absorption of radiation by Rydberg atoms}

Another series of physical effects is expected when one excites $N$ Rydberg atoms in an $\mid \mathrm{nS}>$ state inside the cavity and simultaneously tune the cavity to resonance with the upward transition $\mathrm{nS} \rightarrow \mathrm{nP}$. In this situation, the $\mathrm{B}$ loch vector is initially put in the $\theta=\pi$ position, which is also the only stable permanent position of the vector. No spontaneous irreversible transfer to the other state of the transition can occur, and the only observable effects are then consisting in collective absorption of radiation by the atoms in interaction with the cavity mode.

\subsection{Rabi nutation in Rydberg levels $\left({ }^{6}\right)$}

Let us first fill the cavity mode by an external resonant field of strength $\&_{1}$. The Bloch vector undergoes a Rabi nutation, resulting in a periodic variation of the populations of levels nS and nP, which can be recorded by the "frozen evolution" field ionization technique.

Figure 6: Relative transfer from Teve1 30p to level 30 s as a function of time when a smal1 external microwave field is applied : a) Rabi nutation when the number of excited atoms $N$ is 300 ; b) radiative damping of this nutation. when $N \approx 2000$
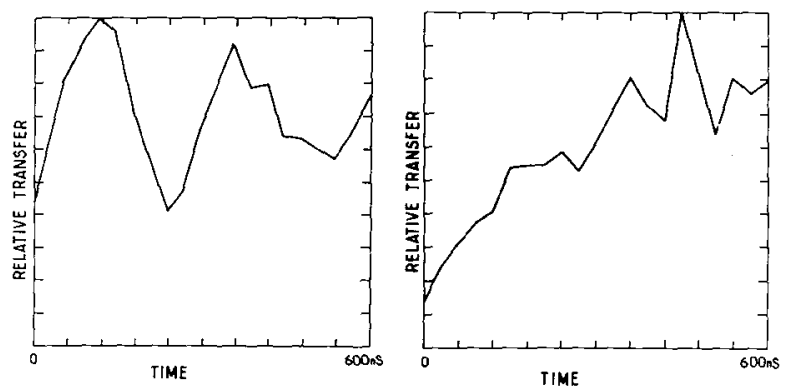
The left part of figure 6 gives as an example the ratio of the population of the $30 \mathrm{P}$ to $30 \mathrm{~S}$ states of sodium as a function of time $t$, when $N \approx 300$ atoms have been excited in the lower 30 s state at time $t=0$. The modulation of the transfer at the Rabi frequency $\nu_{1}=\frac{\mathrm{d} \mathbb{E}_{1}}{\mathrm{~K}}$ is clearly observable. In this example, $\mathbb{E}_{1}=$ $3.710^{-3} \mathrm{~V} / \mathrm{cm}$ and $v_{1}=2.2 \mathrm{MHz}$. Due to the large dipole matrix element $\mathrm{d}$, such a low field, corresponding to only 2000 millimeter photons stored in the cavity, is enough to induce a high frequency Rabi nutation. This kind of experiment is very convenient to calibrate in an absolute way the field in the cavity when one knows the electric dipole matrix elements.

But, when the Bloch-vector is put in the $\theta=0$ position at half the Rabi period, the collective superradiant spontaneous damping towards the $\theta=\pi$ position competes with the Rabi nutation. As a result, when the number $N$ of excited atoms is high enough so that $T_{R}$ is less than the Rabi period $1 / \nu_{1}$, the Rabi oscillation is destroyed by the collective damping. Experimentally, for $N>2000$, the Rabi nutation can be no longer observed (right part of Fig. 6).

This kind of collective damping broadening effects must be avoided when one performs very high resolution spectroscopy in cavities, which restricts the number of excited atoms to be used in a spectroscopy experiment.

With a cavity having a higher finesse, and hence a longer $T_{\text {cav }}$, it will be possible to record similar evolutions corresponding to a very low number of excited atoms interacting with a very smal1 number of photons present in the mode. We hope to be able to eventually observe "single atoms effects" such as those described in $\hat{\S} 3$ of the previous paper.

\subsection{Absorption of very sma1l amount of radiation}

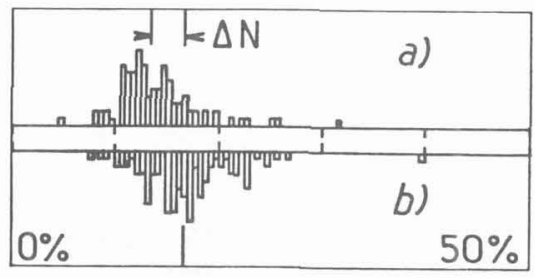

Figure 7 : Histogram of relative transfers from level 30 s to level $30 \mathrm{P} 1 / 2$ : a) without external field; b) with an applied field of $310^{-14} \mathrm{~W}$; an increase $\Delta N$ of the mean value of transfers is clearly appearing.

The previous experiment can be resumed with a strongly attenuated microwave field. Figure $7-b$ gives the histogram of transfers $\Delta N$ from level $30 \mathrm{~S}$ to level $30 \mathrm{p} 1 / 2$ for $N \approx 1200$ atoms and for a microwave power of $0.04 \mathrm{pW}\left(4 \times 10^{8}\right.$ photons $/ \mathrm{s}$ at $134 \mathrm{GHz}$, i.e. 1000 photons impinging on the atoms at each pulse). Figure 7-a gives the same histogram without external microwave. The mean value of the transfers clearly shifts from value $17 \%$ to the value $13 \%$, this last value corresponding to the thermal background at room temperature. The difference $\Delta N=4 \%$ corresponds to 50 photons absorbed by the atoms, which yields a quantum efficiency $n$ of the detection equal to $50 / 1000=5 \%$. Such a quantum efficiency is quite good for this spectral domain and shows the advantage of using Rydberg atoms as mm wave detectors 


\subsection{Collective absorption of blackbody radiation $\left({ }^{7}\right)$}

Another set of experiments has been performed, dealing with the absorption of thermal radiation background by Rydberg atoms. More precisely, we have observed the modification of this absorption when the cavity is tuned to resonance with the $30 \mathrm{~S}^{1 / 2}-30 \mathrm{P} 1 / 2$ transition $(v=134 \mathrm{GHz})$, the $30 \mathrm{~S} 1 / 2$ state being initially populated by the laser. We measured here, by field ionization technique, the populations of $S$ and $P$ levels at the end of the coupled evolution atom + cavity. When the cavity is offresonant, one observes, at room temperature, a thermal transfer of rough $1 y 10 \%$ from $30 \mathrm{~S} 1 / 2$ leve 1 to $30 \mathrm{P} 1 / 2$, corresponding to the absorption of blackbody radiation by the atoms in the modes transverse to the cavity axis and outside the cavity during the drift from the cavity to the detection region (fig. 8-a). When the cavity is tuned to resonance, an increase $\Delta N$ of the number of transferred atoms is observed.
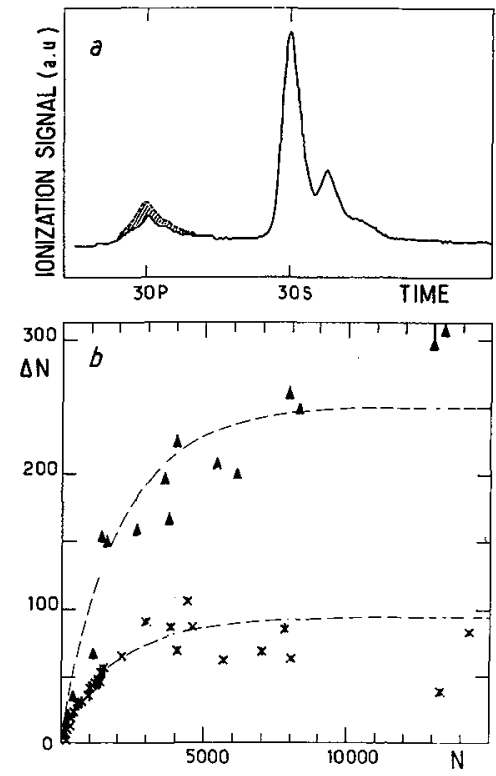

Figure 8 : a) ionization signal showing the variation of population transfer from $30 \mathrm{~S}$ to $30 \mathrm{P}$ level when the cavity is untuned (full line) and tuned to resonance (dashed line). b) variation of transfer $\Delta \mathrm{N}$ as a function of the number $\mathrm{N}$ of excited atoms; crosses : experiment performed at $300^{\circ} \mathrm{K}$; triangles : same experiment with a heated field mode (the experimental uncertainty on $\mathrm{N}$ is around 25\%)

Figure 8-b gives a plot of $\Delta N$, averaged over 300 events, as a function of the total number of excited atoms $N$. We see that, in agreement with the theory of the previous paper, $\Delta N$ saturates to a value of the order of $2 \bar{n}=2 k_{B} T / K \omega$ for large $N$ values.

The lower part of Figure $8-b$ gives the limit

$80 \pm 20$ absorbed photons for the set of 2 two-level systems coexisting in the $30 \mathrm{~S}^{1} / 2 \rightarrow 30 \mathrm{P} 1 / 2$ transition. When a magnetic field is applied in the cavity, in the

way described in paragraph 1.2 , so that a single two-level atom system interacts with the cavity mode, we have checked that the asymptotic value is divided by roughly a factor 2; the formula $\Delta N=g \frac{k T}{h y}$ where $g=2$ or 1 is the degeneracy factor, gives a value in agreement with the measured one, within the error bars. This is an experimental evidence that the system of $\mathrm{N}$ excited atoms behaves as a single collective system since it can absorb only $\frac{k T}{h \nu}$ thermai photons per two level system and not $N / 2$, as would be expected if the atoms were coupled independently from each other to the cavity.

This system provides an absolute thermometer to measure the effective temperature of the cavity mode. The upper part of $F i g .8-b$ has been obtained when this mode was heated by a hot tungsten wire $\left(T \approx 1600^{\circ} \mathrm{K}\right.$ ) coupled to the cavity mode. One sees clearly an increase of the maximum number of transferred atoms $\triangle N=270 \pm 60$ 
(no magnetic field was present in this case), the previous formula with $g=2$ yields a value $\mathrm{T}_{\text {rad }} \gtrsim 900^{\circ} \mathrm{K}$, which means that the radiation reaches a temperature equal to about half the hot wire one, a result consistent with estimates based on simple cavity coupling models.

\section{Conclusion}

This brief survey of experimental results has allowed us to emphasize some striking features of Rydberg states in interaction with an electromagnetic wave in a cavity; in particular it has been shown that the collective radiative decay of the whole sample plays a very important part in different experiments : Rydberg maser of course, but also Rabi nutation in an external field and absorption of blackbody radiation.

\section{References}

( $\left.{ }^{1}\right)$ GOY P., Int. J. of Infrared and Mi11imeter waves, $\underline{3}$ (1982), 221

$\left({ }^{2}\right)$ GROSS M. , GOY P., FABRE C., HAROCHE S., RAIMOND J.M., Phys. Rev. Letters, 43 (1979), 343

$\left({ }^{3}\right)$ MOI L., FABRE C., GOY P., GROSS M., HAROCHE S., ENCRENAZ P., BEAUDIN G., LAZAREFF B., optícs Commun. 33 (1980), 47

$\left({ }^{4}\right)$ MOI L., GOY P., GROSS M., RAIMOND J.M., FABRE C., HAROCHE S., submitted to Phys. Rev. A (1982)

(5) GOY P., MOI L., GROSS M., RAIMOND J.M., FABRE C., HAROCHE S., submitted to Phys. Rev. A (1982)

(6) HAROCHE S., GOY P., RAIMOND J.M., FABRE C., GROSS M., to be pubTished Proc. Roy. Soc. (1982)

(7) RAIMOND J.M., GOY P., GROSS M. , FABRE C., HAROCHE S., Phys. Rev. Letters, $49(1982), 117$. 